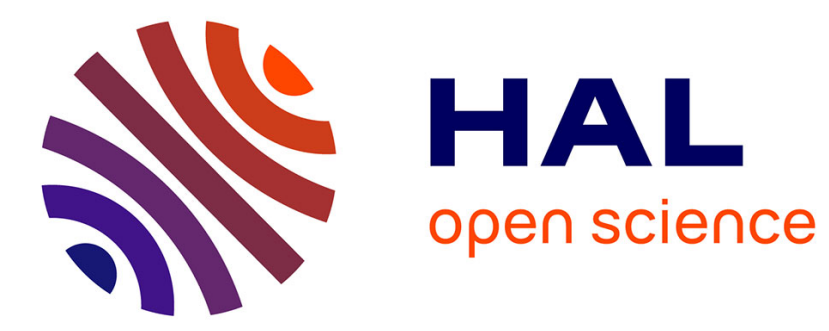

\title{
Le developpement par la rente dans les petites economies insulaires
}

Bernard Poirine

\section{To cite this version:}

Bernard Poirine. Le developpement par la rente dans les petites economies insulaires. Revue Economique, 1993, 44 (6), pp.1169-1199. hal-00974442

\section{HAL Id: hal-00974442 \\ https://hal.science/hal-00974442}

Submitted on 7 Apr 2014

HAL is a multi-disciplinary open access archive for the deposit and dissemination of scientific research documents, whether they are published or not. The documents may come from teaching and research institutions in France or abroad, or from public or private research centers.
L'archive ouverte pluridisciplinaire HAL, est destinée au dépôt et à la diffusion de documents scientifiques de niveau recherche, publiés ou non, émanant des établissements d'enseignement et de recherche français ou étrangers, des laboratoires publics ou privés. 


\section{SciencesPo. Les Presses}

Le développement par la rente dans les petites économies insulaires

Author(s): Bernard Poirine

Reviewed work(s):

Source: Revue économique, Vol. 44, No. 6 (Nov., 1993), pp. 1169-1199

Published by: Sciences Po University Press

Stable URL: http://www.jstor.org/stable/3502273

Accessed: 04/12/2012 23:41

Your use of the JSTOR archive indicates your acceptance of the Terms \& Conditions of Use, available at

http://www.jstor.org/page/info/about/policies/terms.jsp

JSTOR is a not-for-profit service that helps scholars, researchers, and students discover, use, and build upon a wide range of content in a trusted digital archive. We use information technology and tools to increase productivity and facilitate new forms of scholarship. For more information about JSTOR, please contact support@jstor.org. 


\title{
Le développement par la rente dans les petites économies insulaires
}

\author{
Bernard Poirine
}

Quel que soit leur statut politique, les petites économies insulaires sont souvent très dépendantes d'une rente extérieure administrative, militaire, ou privée (envois de travailleurs émigrés). II en résulte des "déséquilibres" souvent stigmatisés : forte dépendance envers "l'aide " extérieure, déficit de la balance commerciale, " hypertrophie » du secteur tertiaire, chômage et/ou émigration.

L'article présente une analyse théorique qui explique ces "déséquilibres" comme une forme du "syndrome hollandais " observé dans les économies de rente énergétique, puis esquisse une théorie de l'échange international de services non marchands, qui permet de rendre compte de l'apparente "spécialisation " des petites économies insulaires dans l'exportation vers de " grands pays " de " services " militaires, géostratégiques, ou géopolitiques non marchands, contre une aide publique qui permet de financer les importations de biens privés.

On appellera ici petites économies insulaires les îles de moins d'un million d'habitants (le plus souvent beaucoup moins). Ces économies, dépendantes politiquement comme les DOM-TOM ou bien indépendantes avec parfois quelques limites de souveraineté comme pour certaines îles dans l'orbite économique de la Nouvelle-Zélande ou des États-Unis, sont « téléguidées », dans le sens où leur économie est « intégrée à distance » à l'économie « tutélaire » qui les prend en charge. Cette forme de développement «impulsé du dehors » par une « rente administrative » présente de grandes similitudes avec celle qui est observée dans des économies de « rente pétrolière » (ou « phosphatière » pour certaines îles-États du Pacifique comme Nauru ou Kiribati), notamment du point de vue de l'existence du "syndrome hollandais" (dutch disease) impliquant l'« éviction » d'activités d'exportation ou concurrentes d'importations, mais aussi avec celle d'une économie régionale de type "ville de garnison », "ville de retraités » ou «banlieue dortoir », intégrée à un ensemble économique de niveau industriel développé.

Dans ce cadre théorique, un modèle de développement, jusqu'ici considéré comme «dépendant» et donc fondamentalement «pervers », peut au contraire se justifier comme répondant à une spécialisation internationale particulière, conforme à la logique de l'avantage comparatif, et donc viable à long terme dans le cadre d'une étroite association à une économie métropolitaine ou dominante, et compatible avec différents degrés possibles de souveraineté pour l'économie insulaire " associée ».

Cette étude aborde la théorie et la pratique des petites économies insulaires dépendantes dont la croissance est impulsée de l'extérieur par une rente publi- 
que (militaire et/ou civile) et/ou par les ressources tirées des envois de travailleurs émigrés.

Seront abordés successivement :

1. La formalisation théorique d'une économie de rente insulaire et ses conséquences pour la politique économique ;

2. L'illustration du modèle dans quelques cas particuliers : les DOM-TOM insulaires des Antilles, du Pacifique et de l'océan Indien, d'une part, les petites îles indépendantes du Pacifique, d'autre part ;

3. Le développement par la rente : sa viabilité et sa logique dans le cadre de l'échange international marchand et non marchand.

\section{L'ÉCONOMIE MIRAGE-ARABE : UNE THÉORIE DE L'ÉCONOMIE DE RENTE INSULAIRE}

Une petite économie insulaire dépendante répond au modèle de développement MIRAGE si elle est caractérisée par :

- une forte émigration et l'importance des envois de travailleurs émigrés pour l'alimentation de la demande interne (MI comme Migration, R comme Remittances, ou remises de travailleurs émigrés à leur famille) ;

- une forte composante d'aide publique extérieure pour le financement des dépenses publiques : aide au développement, coopération, aide budgétaire, ou transferts publics dans le cas d'économies non indépendantes (c'est le A de MIRAGE : AID) ;

- une forte composante de dépenses publiques dans la demande finale, notamment par l'embauche d'une nombreuse bureaucratie administrative (c'est le GE de MIRAGE : Government Expenditure) ${ }^{1}$.

Dans une variante de ce modèle, l'abondance de la rente administrative et le haut niveau des salaires publics et privés du secteur protégé rendent inutile l'émigration vers l'économie tutélaire : celle-ci disparaît, ou plutôt elle est remplacée par une migration interne vers le secteur public « surpayé », même par rapport à la norme métropolitaine : c'est le cas de la plupart des DOM-TOM ${ }^{2}$. Ce modèle de

1. Ce modèle a d'abord été exposé sous le nom MIRAB (avec un B comme bureaucratie) par Bertram et Waters [1985]. Harold Brookfield a, par la suite, suggéré à ces auteurs de remplacer le B de « Bureaucracy » par le GE de « Government Expenditure », le terme de MIRAGE lui semblant plus parlant pour évoquer ce type d'économie.

2. En Polynésie française, un tel mouvement de migration interne vers le secteur « militaire enclavé » a été particulièrement voyant, puisque les agriculteurs et les pêcheurs des archipels se sont précipités en grand nombre à partir de 1962 vers les sites atomiques en construction des Tuamotu, puis vers la base arrière du CEP à Tahiti, attirés par des salaires très élevés pour l'époque, ce qui a stoppé net un mouvement d'émigration vers la Nouvelle-Calédonie et la métropole qui prenait de l'ampleur avant l'arrivée du CEP. La Nouvelle-Calédonie est un cas un peu à part, en raison des ressources apportées par son secteur minier, qui donnent à la rente administrative un rôle relativement moins déterminant que dans les autres DOM-TOM. 
développement peut être résumé sous le sigle ARABE pour : Aide, Rente Administrative (ou Atomique pour la Polynésie française), et Bureaucratie d'État.

\section{Émigration et chômage dans l'économie de rente insulaire}

\section{Les hypothèses du modèle}

On s'inspirera ici d'un modèle proposé par Bertram [1986] pour l'analyse de petites économies insulaires du Pacifique dépendant économiquement de la Nouvelle-Zélande, lui-même inspiré de celui que Corden et Neary [1982] avaient utilisé pour l'analyse de la désindustrialisation provoquée par un boom minier dans une petite économie ouverte.

1. On suppose une petite économie en développement ouverte (n'ayant aucune influence sur les prix internationaux des produits qu'elle importe ou exporte), présentant une faible productivité du travail, en étroite relation avec une économie industrielle, à haute productivité du travail et salaire réel plus élevé, vers laquelle l'émigration ne rencontre pas d'obstacle légal.

2. Cette petite économie en développement comporte trois secteurs :

- un secteur traditionnel «villageois » d'autosubsistance produisant également des exportations agricoles « traditionnelles »;

- un secteur public des services non marchands ;

- un secteur privé capitaliste produisant des biens et services pour la demande locale ou pour l'exportation.

3. Le marché du travail est ouvert. Il n'y a aucune restriction à l'émigration, rendue facile par l'appartenance politique à un ensemble métropolitain (DOMTOM, Guam, Samoa américaines) ou par des accords étroits avec l'ancienne puissance métropolitaine continuant à accorder aux insulaires les avantages de la citoyenneté (cas des îles Cooks avec la Nouvelle-Zélande).

4. Il y a parfaite mobilité du facteur travail entre les trois secteurs insulaires et entre ces secteurs et le secteur « étranger »d'autre part.

\section{Exposé géométrique du modèle}

On s'intéressera ici à la répartition de la main-d'œuvre entre les trois secteurs et à la manière dont l'accroissement de la population active pourra trouver à s'employer sur place ou à l'extérieur de l'économie insulaire.

Sur le graphique 1, l'axe horizontal représente la quantité totale de maind'œuvre disponible dans l'île exprimée en temps de travail. L'axe vertical représente le taux de salaire réel, mesuré en unités d'un « panier représentatif » de produits.

$\mathrm{W}^{*}$ est le taux de salaire réel moyen « étranger » attendu en cas d'émigration, net des coûts de transport, des coûts psychologiques de l'émigration, et du 
risque de ne pas trouver de travail dans l'économie tutélaire. Pour simplifier, nous appellerons désormais $\mathrm{W}^{*}$ le «salaire d'émigration ». Compte tenu des corrections ci-dessus évoquées, le salaire d'émigration sera sensiblement inférieur au salaire moyen dans l'économie tutélaire, ce qui implique en particulier que même si le SMIG de l'économie micronésienne est inférieur à celui de l'économie tutélaire de $20 \%$ ou $30 \%$ en valeur réelle, cette différence peut être en fait insuffisante pour motiver un courant d'émigration.

Le revenu réel de l'activité non salariée dans le secteur traditionnel d'autosubsistance est donné dans la partie (A) par la courbe Ls (indice $s$ pour "subsistance »), où la quantité de travail employée est mesurée sur l'axe horizontal dans le sens 0 '0, donc de droite à gauche. La forme de la courbe s'explique par la loi des rendements décroissants : à droite de A la courbe a une branche verticale, ce qui exprime qu'il y a un minimum irréductible de force de travail dans ce secteur, en dessous duquel le mode de vie villageois traditionnel disparaît ${ }^{1}$. À gauche, la courbe exprime des rendements constants jusqu'à 0 , avec un revenu réel d'auto-subsistance W1 qui reste inférieur au salaire d'émigration $\mathrm{W}^{*}$.

Dans le cadrant $(\mathrm{C})$, la courbe $\mathrm{L}_{m}$ représente la demande de travail du secteur " capitaliste " (indice $m$ pour "manufacture »), mesurée dans le sens habituel 00 '. Elle obéit aussi à la loi des rendements décroissants, et exprime que, au taux de salaire d'émigration $\mathrm{W}^{*}$, très peu d'activités pourraient être rentables localement, en raison du handicap occasionné par la faiblesse des économies d'échelle dans la production pour un marché très réduit. Ce secteur n'offre donc que peu de travail si le taux de salaire réel s'aligne sur le taux de salaire d'émigration $W^{*}$ d'un pays industrialisé, et encore moins s'il s'aligne sur un niveau de traitement public $\mathrm{W}_{g}$ supérieur à $\mathrm{W}^{*}$. L'emploi dans ce secteur peut croître au-dessus du minimum OC si le taux de salaire baisse nettement en dessous de $\mathrm{W}_{g}$ et $\mathrm{W}^{*}$, les activités de substitution d'importation ou d'exportation devenant alors plus compétitives, malgré la productivité du travail plus faible que dans l'économie tutélaire. En dynamique, la courbe $\mathrm{L}_{m}$ se déplace vers la droite à mesure que les dépenses publiques augmentent, en raison de l'effet multiplicateur de celles-ci sur la demande au secteur privé protégé de la concurrence des importations (services, BTP).

La courbe $\mathrm{L}_{g}$ (cadrant $\mathrm{B}$ ) représente la demande de travail du secteur public (indice $g$ pour " gouvernement»), représentée dans le sens habituel 00 '. Chez Bertram, elle présente une élasticité unitaire, ce qui reflète le fait qu'avec une masse salariale publique constante MS, le nombre d'emplois créés $\mathrm{L}_{\mathrm{g}}$ dépend

1. Ou, si l'on préfere, la production du bien public socio-culturel « qualité de vie insulaire communautaire » ne peut subsister en dessous d'un certain degré de participation au mode de production villageois traditionnel, et si la communauté désire préserver ceci, elle devra offrir à ceux qui restent dans ce secteur des compensations monétaires (envois de fonds) ou non monétaires (plus de loisirs) telles que ceux-ci choisiront de rester, malgré un revenu réel inférieur au taux de salaire « d'émigration ». 
Graphique 1. Modèle à trois secteurs d'une économie micronésienne " téléguidée "

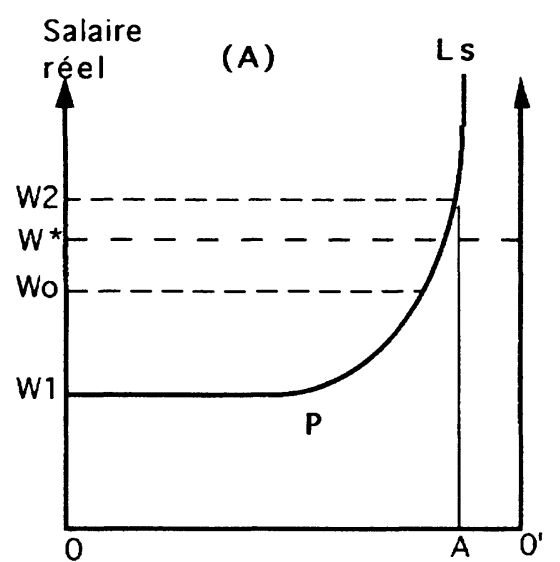

Nombre d'emplois

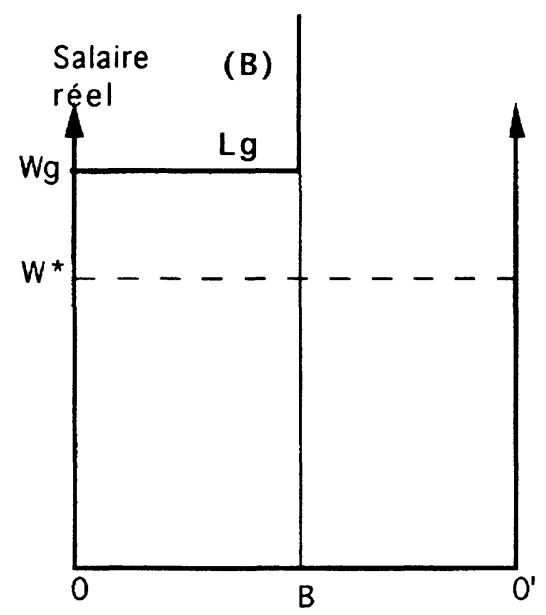

Nombre d'emplois

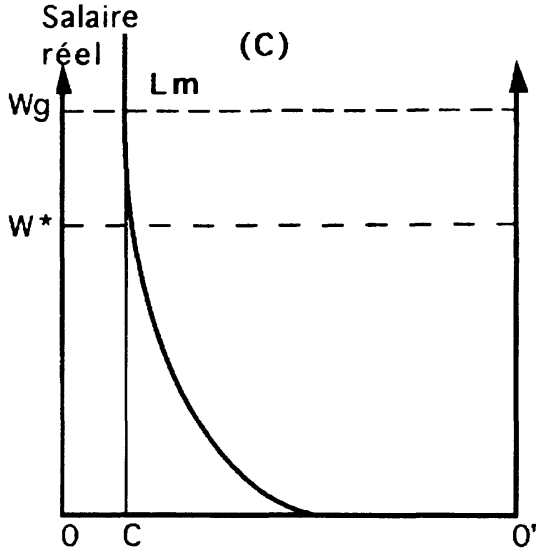

Nombre d'emplois

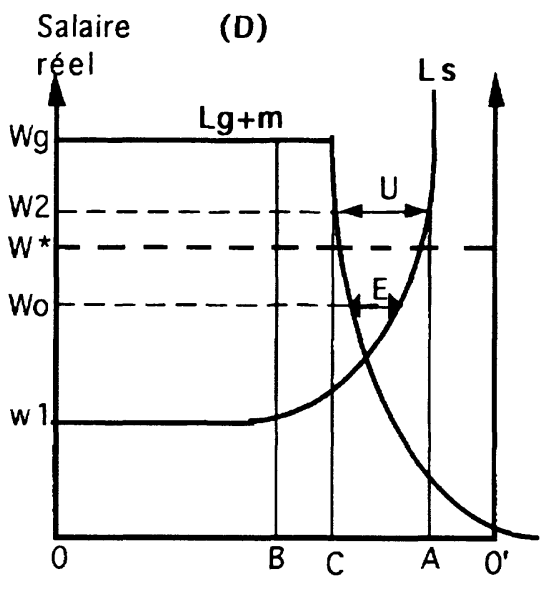

Nombre d'emplois

du traitement moyen versé aux fonctionnaires $\mathrm{W}_{g}$ de la façon suivante: $\mathrm{MS}=\mathrm{W}_{g} \cdot \mathrm{L}_{g}=$ constante. Dans le cas des DOM-TOM français, il vaut mieux remplacer cette hypothèse par celle d'un salaire public « exogène ", correspondant au niveau moyen de traitement public «métropolitain », majoré d'un « coefficient » qui varie selon les cas (+ $84 \%$ en Polynésie française par exemple). Cela implique une courbe $\mathrm{L}_{g}$ dont la forme est horizontale à un niveau de salaire supérieur au niveau métropolitain $\left(\mathrm{W}_{g}>\mathrm{W}^{*}\right)$, jusqu'à ce que la masse salariale publique disponible soit épuisée, ce qui correspond à un niveau d'emplois publics $\mathrm{OB}$, au-delà duquel la courbe devient verticale. 
Dans le cadrant (D), les courbes $\mathrm{L}_{g}$ et $\mathrm{L}_{m}$ sont additionnées horizontalement, ce qui donne une courbe $\mathrm{L}_{g}+m$ exprimant la demande de travail dans les secteurs public et capitaliste.

\section{Les conséquences du modèle}

Dans ces conditions, la courbe $\mathrm{L}_{g}+m$ du cadrant $\mathrm{D}$ a la forme indiquée, la distance horizontale entre celle-ci et la courbe $\mathrm{L}_{s}$ mesure l'excédent de maind'œuvre inemployée dans l'économie insulaire, qui va se traduire par du chômage ou de l'émigration.

Ainsi, si le niveau de salaire dans le secteur capitaliste est $\mathrm{W}_{2}$, supérieur au salaire d'émigration $\mathrm{W}^{*}$, les secteurs capitalistes et publics absorbent ensemble la quantité de main-d'œuvre OC, tandis que le secteur traditionnel d'autosubistance absorbe la quantité O'A.

Le surplus de main-d'œuvre qui ne trouve pas à s'employer sur place est donc CA. Ce surplus se traduit par du chômage (indiqué par la double flèche $U$ dans le cadrant D), car le salaire d'émigration n'incite pas à partir trouver du travail à l'extérieur. Ce chômage peut être réduit dans la mesure où le secteur d'autosubsistance a la possibilité d'offrir des occupations moins rémunérées que le salaire minimum du secteur capitaliste, mais néanmoins préférables au " chômage sec » d'une économie développée sans secteur informel développé. Il est par contre strictement égal à $U$ si le salaire minimum est identiquement appliqué dans tous les secteurs, y compris le secteur traditionnel, ou si un système de revenu minimum (UNEDIC, RMI) apporte au chômeur un niveau de vie supérieur à ce qu'offrirait le secteur traditionnel dans la pire des hypothèses, à savoir le salaire réel de subsistance minimum $\mathrm{W}_{1}$.

Par contre, si le salaire moyen du secteur privé est inférieur au salaire d'émigration $\left(\mathrm{W}_{\mathrm{o}}<\mathrm{W}^{*}\right)$, l'excédent de main-d'œuvre sera moindre d'une part (mesuré par la double flèche $E$ dans le cadrant $D$ ), et d'autre part se traduira en grande partie par l'émigration vers l'économie industrielle tutélaire, plutôt que par le chômage.

Le niveau du chômage dépendra en dynamique du rythme d'accroissement de la population active (segment OO'), de celui des emplois publics (déplacement de la courbe $\mathrm{L}_{g}$ ), qui détermine celui du déplacement de la courbe $\mathrm{L}_{m}$ vers la droite (effet multiplicateur). Le chômage peut être limité si le SMIG est maintenu assez bas, ce qui va, d'une part, limiter l' « effet d'écrémage » sur le secteur traditionnel et, d'autre part, encourager l'émigration ${ }^{1}$. Il peut aussi être évité,

1. En Polynésie française, le niveau du SMIG (4 $400 \mathrm{FF}$ en 1991) est inférieur à celui du SMIC métropolitain (5 $400 \mathrm{FF}$ ), mais la différence n'est sans doute pas assez importante pour compenser le coût du voyage (presque trois SMIG), le coût psychologique, l'incertitude née du chômage important en métropole qui réduit l'espérance mathématique du gain, etc. Le SMIG horaire est de 4,52 \$ US en Polynésie française depuis 1989, contre 3,80 \$ US aux États-Unis en avril 1990 (et 4,25 \$ depuis avril 1991). Aux Samoa américaines, le SMIG est de 2,17 \$ en 1991. Le SMIG en Polynésie française a augmenté de $50 \%$ en 1978, et a doublé entre 1977 et 1988. 
malgré un SMIG élevé par rapport au niveau de salaire d'émigration (impliquant une émigration nulle), si le déplacement du « coude » de la courbe $\mathrm{L}_{g}$ vers la droite dû aux créations d'emplois publics est particulièrement rapide.

L'effet d'éviction de la rente publique sur les autres secteurs (traditionnel et capitaliste) est d'autant plus grand que l'écart positif entre les rémunérations du secteur public et du secteur privé est important, même si en dynamique l'effet mulitiplicateur de l'accroissement des transferts publics provoque un déplacement de $\mathrm{L}_{m}$, donc des créations d'emplois privés dans le secteur protégé (commerce, services, BTP), du moins tant que le niveau de la rente publique augmente. Le haut niveau des salaires du secteur privé capitaliste, «tiré » par celui des salaires du secteur public, impose des techniques « capital intensive » peu favorables à la création d'emplois, en dépit d'un niveau de productivité moyen relativement bas dans le secteur traditionnel comme dans le secteur public.

Lorsque la rente publique stagne, la courbe $\mathrm{L}_{g}$ ne se déplace plus vers la droite, seule l'arrivée d'autres ressources extérieures (tourisme, envois de travailleurs émigrés, exportations de minerais), ou bien la baisse des salaires publics, peuvent permettre des créations d'emplois. Mais dans une économie insulaire à haut pouvoir d'achat, au SMIG comparable (après correction pour coûts psychologiques et de transport) à celui de l'économie « métropolitaine ", il n'est pas aisé de trouver des ressources extérieures susceptibles de remplacer la rente administrative ou militaire publique, car la compétitivité des activités d'exportation (y compris le tourisme) est compromise par le niveau élevé des coûts salariaux, comparé à celui d'autres économies insulaires moins favorisées par la rente administrative ou militaire. Ce handicap est souvent renforcé par une surévaluation artificielle de la monnaie insulaire rendue possible, malgré un fort déficit de la balance des paiements courants, par l'intégration à la «zone monétaire » de l'économie tutélaire.

Par ailleurs, dans cette conjoncture, l'existence d'un niveau "exogène " élevé des salaires dans la fonction publique limite la capacité d'absorption de celle-ci, puisqu'un plus bas niveau moyen des traitements permettrait d'embaucher plus pour une même enveloppe budgétaire, ce qui est politiquement impossible parce que les fonctionnaires « locaux » exigent un « alignement » sur les salaires des fonctionnaires «expatriés » de niveau équivalent, bénéficiant d'un traitement «majoré » pour cause d' $d$ éloignement » de la métropole.

Dans les économies insulaires à faible pouvoir d'achat, ayant acquis leur indépendance avant que le secteur public ait aligné le niveau de ses salaires par référence à celui de l'ancienne métropole, le bas niveau des traitements du secteur public permet, grâce à l'aide budgétaire de l'ancienne métropole, d'embaucher une fraction importante de la population active restée sur l'île. Par ailleurs, le niveau relativement bas des salaires dans le secteur capitaliste local, comparé à celui du salaire d'émigration attendu, incite une forte proportion des jeunes actifs à émigrer vers l'économie industrielle tutélaire.

Ces derniers reversent à leur famille une partie de leur salaire. Ces envois constituent un appoint considérable pour la demande qui s'adresse au secteur 
capitaliste local, notamment pour le secteur de l'importation et de la construction. Au terme de cette évolution, le secteur moderne devient le secteur étranger, c'est-à-dire que les salaires du secteur à haute productivité proviennent de l'étranger, sous forme d'envois de travailleurs émigrés, ce qui permet d'assimiler l'économie insulaire à une «banlieue dortoir » de l'économie tutélaire : les activités subsistantes dans l'île sont celles qui relèvent d'un secteur traditionnel "subventionné » par les mandats des émigrés, d'un secteur public bénéficiaire de l'aide publique étrangère, et d'un secteur capitaliste "naturellement » protégé de la concurrence (commerce, importation, services financiers, Bâtiment et Travaux publics, maintenance, entretien et réparations). Aide publique et envois d'émigrés permettent d'équilibrer la balance des paiements malgré un fort déficit des échanges commerciaux.

\section{L'effet d'éviction des activités productives par la rente administrative : une forme de dutch disease}

L'analyse des effets de la rente pétrolière, énergétique (gaz naturel), ou minière (dans le cas de l'Australie) a donné lieu à une littérature théorique qui n'est pas sans intérêt quand il s'agit d'analyser les effets d'autres formes de rente sur une économie.

Le débat s'est notamment consacré à l'analyse de ce qu'il est convenu d'appeler le dutch disease (syndrome hollandais). On constate, en effet, dans les pays disposant brusquement d'une rente «naturelle» des phénomènes communs : éviction des activités consacrées à des biens ou services exportables, hausse des prix dans le secteur protégé de la concurrence internationale (services notamment) et du niveau de prix relatif des produits de ce secteur par rapport à ceux du secteur exposé, hypertrophie du secteur tertiaire et «désindustrialisation» de l'économie; enfin, surévaluation de la monnaie nationale. À un degré plus ou moins grave, ces phénomènes ont été observés aux Pays-Bas (gaz naturel), au Royaume-Uni (pétrole de la mer du Nord), en Australie ( «boom minier ») ${ }^{1}$ et, bien entendu, dans les pays exportateurs de pétrole du Moyen-Orient, ainsi que dans certaines économies insulaires productrices de pétrole comme Trinidad, ou de phosphate comme Nauru.

Plusieurs auteurs ont tenté de montrer comment ces phénomènes résultent de la réallocation des ressources dans l'économie suite à un bouleversement des prix relatifs de ces ressources provoqué par l'arrivée d'une rente naturellle.

L'analyse présentée ici, adaptée d'un modèle d'équilibre général à deux secteurs proposé par S. Alam [1982] pour les économies de rente pétrolière, repose sur la distinction entre un secteur fabriquant les biens et services échangés (traded goods), ou secteur « exposé » et un secteur produisant les biens et services non échangés (non traded goods), donc secteur " protégé ». Celui-ci est défini

1. M. Corden, J.-P. Neary [1982], p. 825-848. 
comme celui des services (à l'exception du tourisme international, exposé à la concurrence internationale), mais comprend aussi une partie du secteur secondaire : bâtiment et travaux publics, monopole de l'électricité, autres monopoles «naturels», ou bien encore activités protégées de la concurrence des importations par de très fortes barrières douanières (c'est souvent le cas dans l'agro-alimentaire par exemple : brasseries, charcuteries, produits lactés...).

Soit $\mathrm{A} \mathrm{C}_{0} \mathrm{~B}$ la frontière des possibilités de production (graphique 2) décrivant les combinaisons efficaces de produits des deux secteurs qu'il est possible d'obtenir en faisant le meilleur emploi possible des ressources existantes, avant la rente.

L'arrivée de cette dernière fournit des devises étrangères avec lesquelles la collectivité qui perçoit la rente va pouvoir obtenir " gratuitement » un supplément de produits échangés A A'. La nouvelle frontière des possibilités de production se déplace donc en $\mathrm{AA}^{\prime} \mathrm{C} 2 \mathrm{C} 1 \mathrm{D}$, courbe obtenue par une translation horizontale de longueur AA' de la frontière AQCOB.

En supposant des courbes d'indifférence collectives « normales » ${ }^{1} \mathrm{I}_{0}, \mathrm{I}_{1}, \mathrm{I}_{2}$, les points $\mathrm{C}_{0}$ et $\mathrm{C}_{2}$ représentent respectivement les niveaux de consommation des produits des deux secteurs avant et après la rente. Cependant, le niveau de production correspondant à $\mathrm{C}_{2}$ est $\mathrm{Q}$, où la distance horizontale $\mathrm{QC}_{2}$ est égale à AA', car, dans le secteur des biens échangés, la quantité AA' est importée grâce à la rente.

On constate que dans le cas «normal », le niveau de production du secteur des biens échangés (correspondant à $\mathrm{Q}$ sur l'axe horizontal) baisse par rapport au niveau initial (correspondant à $\mathrm{C}_{0}$ sur l'axe horizontal).

Il y a donc "éviction » de la production agricole ou industrielle du secteur des produits échangés (ou secteur "exposé »), au profit de celle du secteur "protégé », des produits non échangés (dont les services, hors tourisme international), les ressources (travail et capital) allant vers les activités plus rentables, et offrant les meilleurs salaires, du secteur protégé offrant les produits non échangés.

La raison de cet « effet d'éviction » néfaste à l'agriculture et à l'industrie du secteur exposé est simple : l'abondance relative des produits échangés permise par la rente a diminué leur prix relatif par rapport aux produits non échangés. Cela va entraîner l' « effet-prix », c'est-à-dire le mouvement de $C_{1}$ à $C_{2}$ pour la consommation, et de $\mathrm{C}_{0}$ à $\mathrm{Q}$ pour la production : des ressources (capital et travail) vont quitter le secteur «exposé » (produits échangés) pour le secteur «protégé » (produits non échangés), en raison de la hausse du prix relatif des

1. On se sert ici de courbes d'indifférence collectives pour représenter de façon commode (dans ce type de graphique) les courbes de demande agrégées, en ignorant provisoirement le fait bien connu que des changements dans la distribution du revenu des facteurs provoqués par un déplacement le long de la courbe des possibilités de production peuvent affecter la forme de ces courbes de demande agrégées. 
produits de ce dernier secteur, qui élève la valeur de la productivité marginale des facteurs y afférents. Il en résulte une diminution de la production de biens du secteur «exposé », et une hause de la production des biens du secteur " protégé » (déplacement de $\mathrm{C}_{\mathrm{o}}$ à $\mathrm{Q}$ ) : c'est l'explication du dutch disease dans les économies « pétrolières », mais aussi de l'effet d'éviction par la rente administrative dans les microéconomies insulaires.

Graphique 2. Frontière des possibilités de production dans une économie de rente à deux secteurs : produits échangés, produits non échangés.

\section{SNE}

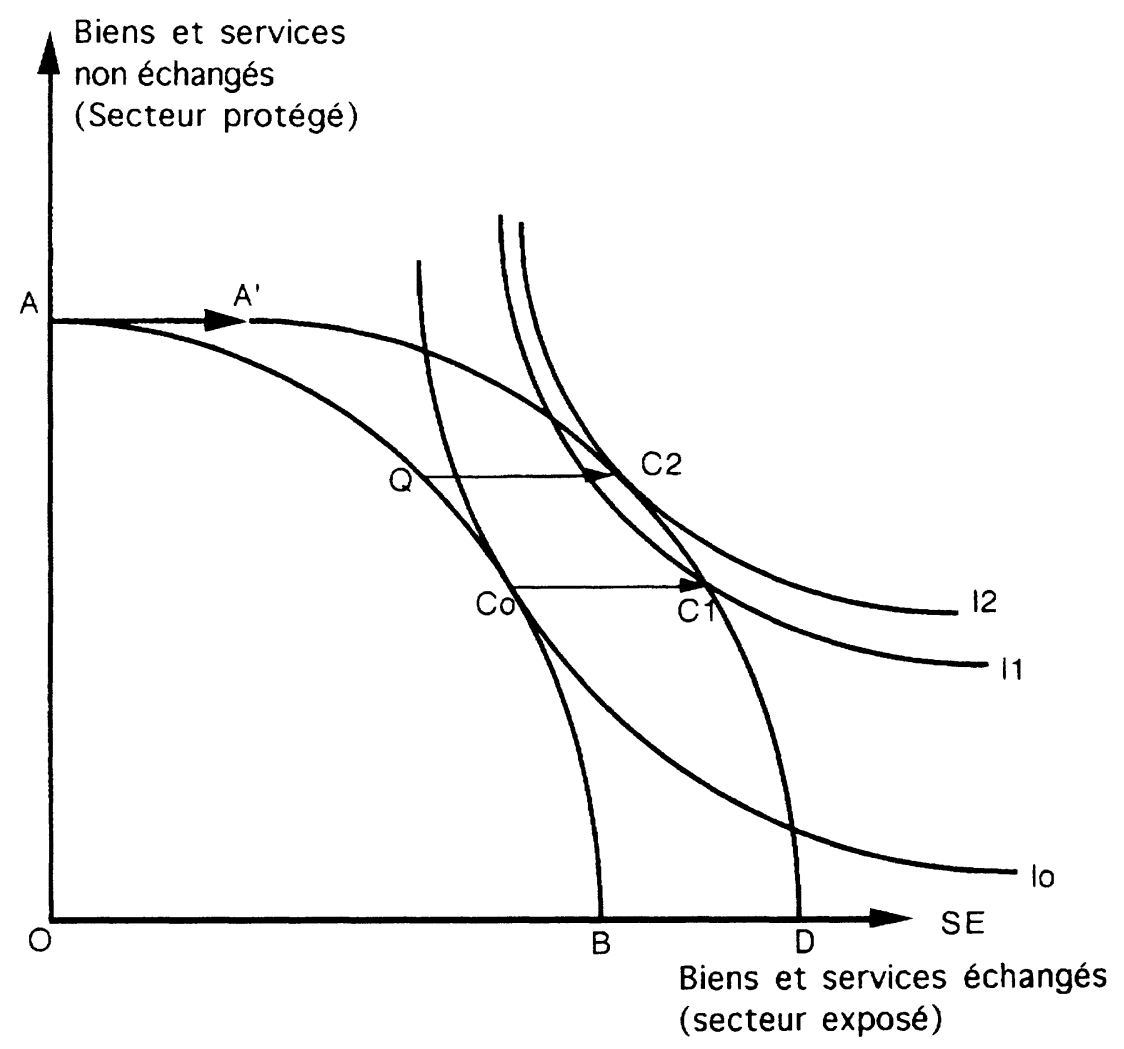

Il faut noter que la baisse de la production du secteur "exposé " ne signifie pas forcément celle du secteur secondaire. En effet, à mesure que l'effet d'éviction s'accentue, le contenu des activités de ce secteur change : les activités d'exportation disparaissent et celles concurrentes d'importations sont de plus en plus protégées par de fortes barrières douanières, alors que se développent surtout les activités protégées naturellement de la concurrence des importations (monopoles énergétiques, bâtiment et industries connexes, maintenance, réparation et entretien). Il en est de même pour le secteur primaire : les cultures d'exportation disparaissent, tandis que se développent les cultures maraîchères ou fruitières mises à l'abri des importations par des contingentements ou de forts droits de douane, destinées à une clientèle fortunée bénéficiaire de la rente. 


\section{Conséquences pour les politiques économiques}

La conclusion de l'analyse théorique ci-dessus est que l'adaptation d'une économie à l'existence d'une rente administrative est semblable à celle qui résulte de la rente pétrolière : l'équilibre qui en résulte peut être qualifié d'optimal, au sens où il reflète l'adaptation naturelle au nouveau prix relatif des ressources, alors que ces économies de «transfert», comme les économies pétrolières, sont souvent stigmatisées comme «déséquilibrées », ou «pathologiques » en raison de l'hypertrophie du secteur tertiaire et des administrations et du profond déséquilibre de leurs échanges extérieurs. La tendance naturelle des experts est invariablement de recommander un "réquilibrage » économique en faveur des secteurs « productifs », dont on cherche à favoriser le développement par différents dispositifs incitatifs (subvention, défiscalisation, etc.). Il en est ainsi de la « loi de défiscalisation » en faveur des investissements dans les secteurs « productifs » des DOM-TOM, encore appelée « loi PONS ».

Une autre politique d'aide consiste à subventionner le secteur d'autosubsistance et d'exportation agricole (de même que la CEE subventionne son agriculture), par exemple sous la forme de subvention de prix agricoles (ce que fait la Polynésie française pour le coprah, payé au producteur six fois le prix de vente à l'exportation).

Des raisons stratégiques à long terme plaident en faveur d'un maintien d'une autosubsistance alimentaire : de même que dans les pays de la CEE ou les pays bénéficiant de la rente pétrolière, il paraît nécessaire de maintenir des activités agricoles par des subventions, même si elles ne sont pas profitables à court terme, afin d'acheter ainsi une assurance pour l'avenir, au cas où les autres sources de revenus (aide internationale, envois de fonds des émigrés), fléchiraient. En tout cas, il semble plus souhaitable de favoriser des schémas de subvention directe des activités agricoles villageoises, plutôt que d'accorder des subventions budgétaires qui ne servent qu'à l'expansion de la bureaucratie au détriment d'activités d'autosubsistance ${ }^{1}$.

Faut-il obligatoirement considérer comme allant de soi les coûteuses politiques de subventions aux secteurs «productifs», ou bien accepter le «déséquilibre » économique résultant des transferts comme une adaptation « optimale » des ressources de l'économie locale à la rente administrative ?

Le même type de question s'est posé aux économies frappées par le dutch disease, dans les pays industrialisés ou en voie de développement bénéficiant d'une rente énergétique ou minière et connaissant de ce fait un mouvement de "désindustrialisation », ou de baisse de la production agricole traditionnelle (Pays-Bas, Royaume-Uni, Australie, Nigéria) : faut-il s'efforcer de « subventionner » les secteurs industriels et agricoles non protégés de la concurrence

1. « Merely pouring more money into local government tends to increase the bureaucratization of Island sociéty, and may threaten the viability of the village mode of production. » (Bertram, op. cit., p. 819.) 
internationale? En supposant que le progrès technique provient en partie d'un phénomène d'apprentissage (learning by doing), il est possible de démontrer théoriquement qu'à long terme un pays peut avoir intérêt à atténuer par des subventions l' «effet d'éviction » de la rente énergétique ou minière sur le secteur industriel et agricole, dans le but d'éviter une contraction du revenu national après l'épuisement des réserves naturelles.

Cependant, cette conclusion ne semble pas être confirmée dans le cas où s'offre la possibilité de capitaliser des réserves en devises, dont le placement permet de constituer un fond de réserve susceptible de prendre le relais de la rente énergétique ou minière à l'issue de son épuisement (l'île de Nauru a ainsi constitué un fonds commun de placement destiné à fournir des revenus financiers susceptibles de prendre le relais des revenus de l'exploitation du phosphate). En effet, dans ce cas, Van Wijnbergen ${ }^{1}$, utilisant un modèle à deux périodes d'une économie ouverte, montre que la justification théorique de subventions au secteur exposé non pétrolier n'est plus aussi assurée. La collectivité peut avoir, dans ce cas, intérêt à se « spécialiser » dans la gestion de la rente financière capitalisée pendant la période d'exploitation de la ressource naturelle, alors que toute politique de subvention aux secteurs agricoles ou industriels pendant cette période diminuerait les sommes capitalisées pour assurer une rente financière perpétuelle aux générations futures.

Enfin, une troisième politique consiste à revenir à une économie d'autosubsistance, ce qui implique une diminution des subventions budgétaires finançant la bureaucratie, une limitation de l'émigration, de telle manière que le chômage créé par le surplus de main-d'œuvre fasse descendre les salaires en dessous du taux de salaire étranger $W^{*}$ jusqu'à $W_{1}$, et peut être une politique d'aide technique pour la modernisation des techniques agricoles. C'est la politique d'autosuffisance, ou d'autarcie. Bertram [1986] remarque justement qu'une telle politique serait extrêmement coûteuse socialement et psychologiquement dans les îles ayant déjà développé des habitudes de consommation de produits importés. La baisse du niveau de vie ne pourrait être justifiée politiquement en l'absence d'un écroulement de la rente (aide et envois de fonds). Pratiquement donc, une telle politique ne peut se justifier que dans les îles les moins avancées du Pacifique qui désirent préserver un système communautaire traditionnel pour des raisons socio-culturelles, et qui seraient suffisamment peu ouvertes au tourisme, aux media et à l'émigration pour que l' "effet de démonstration » ne vienne pas anéantir les efforts d'autosuffisance du gouvernement et augmenter la frustration des habitants et le désir d'émigration.

Du point de vue de la stratégie de développement à adopter, contrairement à ce qui peut se produire dans d'autres PVD non insulaires ou de grande taille, la grande ouverture du marché du travail comme du marché des biens dans les économies inuslaires compromet la rentabilité des productions locales en raison

$\rightarrow$ Sweder Van Wijnbergen, « The Dutch Disease: a Disease After All ? », The Economic Journal, 945, mars 1984, p. 41-55. 
du niveau élevé des salaires qui résulte de la facilité de l'émigration vers les pays « riches »: le niveau moyen plus bas de la productivité des facteurs ${ }^{1}$ ne peut permettre de lutter contre les produits des pays riches librement importés (sauf très forte protection douanière), ce qui condamne la substitution d' importation, et le niveau plus élevé des salaires moyens ne permet pas de lutter contre les produits des PVD à bas salaires (notamment pour les activités de substitution d'exportation, de type zone franche industrielle d'exportation, et pour les exportations de produits agricoles comme le coprah, le café, la vanille). L'effet d'éviction des activités productives qu'on observe ici est dû au niveau des salaires fuxé de façon exogène par rapport à l'économie locale, ou plutôt sans rapport avec le niveau de la productivité du facteur travail. Il en résulte des créations d'emplois réduites dans le secteur capitaliste moderne, sauf cas exceptionnels (investissements extérieurs massifs dans l'industrie minière, ou dans le tourisme notamment, effet multiplicateur de transferts publics extérieurs croissant rapidement sur le secteur tertiaire et secondaire protégé de la concurrence internationale par des barrières naturelles ou douanières).

Dans une telle situation, ni la substitution d' importation, ni la promotion des exportations de produits manufacturés ne peuvent être envisagées comme stratégie de développement viable, en raison de la faible compétitivité des productions locales: salaires de pays riches et productivité de pays pauvres sont compatibles momentanément dans une économie de rente, mais incompatibles à long terme avec le développement des secteurs productifs exposés à la concurrence étrangère. Seules des activités offrant une « rente naturelle », c'est-à-dire basée sur l'exploitation d'un facteur rare sur le plan mondial, sont susceptibles de se développer alors avec profit en fournissant les ressources extérieures qui impulsent la croissance des secteurs protégés : pétrole, phosphate, nickel, perle noire, tourisme "haut de gamme» spécifique, accueil de fonctionnaires « métropolitains », retraités à retraite « majorée », paradis fiscal, activités financière off-shore, productions philatéliques et numismatiques, droits de pêche.

1. En raison des déséconomies d'échelle, des coûts d'approvisionnement en intrants et biens d'équipement, de l'absence d'effets externe de proximité, de la faible éducation de la main-d'œuvre, de la pénurie de cadres, etc. 


\section{LE DÉVELOPPEMENT PAR LA RENTE EN PRATIQUE : QUELQUES ILLUSTRATIONS DU MODĖLE}

\section{L'économie MIRAGE dans les petites économies insulaires du Pacifique}

Beaucoup de petites économies insulaires vivent en partie ou en totalité d'une rente, soit naturelle (exploitation d'une ressource naturelle bénéficiant d'un prix élevé sur le marché mondial : phosphate, vente de droits de pêche), soit monétaire : recettes induites par une base militaire, aide internationale ou bilatérale, notamment en provenance d'une métropole ou d'une ancienne métropole avec qui subsistent des liens privilégiés. La plupart des économies micronésiennes du Pacifique, en particulier, bénéficient d'un niveau élevé d'aide internationale par tête, ce qui leur permet, dans bien des cas, d'entretenir un déficit chronique de leur balance commerciale :

" À l'exception près de Nauru [riche de la rente "phosphatière"], l'aide extérieure est présente dans tous les pays indépendants de l'Océanie insulaire. En 1980, elle équivalait déjà au tiers du PIB aux Samoa américaines, aux Salomon, aux Tonga, à près de la moitié de celui des îles Cook et des Samoa occidentales et à la presque totalité de celui du Kiribati. Au Vanuatu, à Tuvalu, à Niue, à Tokelau et en Micronésie américaine l'aide extérieure s'avérait même supérieure au PIB $^{1}$. »

Le montant de l'aide par habitant est d'autant plus élevé en général que la population est faible, et l'aide est en général plus forte pour les territoires $(649 \$$ par habitant en moyenne) que pour les États indépendants ( $292 \$$ par habitant en moyenne). Elle est bien plus forte pour les îles ( $283 \$$ ) que pour l'ensemble des PVD non exportateurs de pétrole (14 \$ par habitant).

Tous les pays insulaires du Pacifique Sud ont un déficit de leur balance commerciale, comme le montre le tableau 1.

Les îles Cook, Niue et Tuvalu sont des États « librement associés » à la Nouvelle-Zélande, théoriquement indépendants, mais en fait très liés à l'ancienne Métropole. Les citoyens gardent la nationalité néo-zélandaise, les marchandises circulent en franchise de droits vers la Nouvelle-Zélande, les relations extérieures et la Défense sont prises en charge par ce pays. Tokelau est resté une dépendance de la Nouvelle-Zélande.

Ces îles reçoivent une aide très importante, par habitant, et en pourcentage du PNB, puisque l'aide publique dépasse leur PNB. Aux îles Cook, l'aide extérieure s'est accrue de $227 \%$ entre 1977 et 1982.

1. Jean-Pierre Doumenge, «Les bases concrètes de la géopolitique du monde insulaire océanien », Journal de la société des océanistes, 87 (2), 1988, p. 73-88. 
Tableau 1. Déficit de la balance commerciale des pays insulaires du Pacifique Sud

\begin{tabular}{|c|c|c|c|}
\hline En $\$$ australien & $\begin{array}{c}\text { Déficit } \\
\text { comm. } \\
1989 \\
\text { milliers de A } \$\end{array}$ & $\begin{array}{c}\text { Population } \\
1990\end{array}$ & $\begin{array}{c}\text { Déficit } \\
\text { par habitant } \\
\text { en } \$ \text { australien }\end{array}$ \\
\hline Polynésie française ..................... & -874786 & 196300 & -4456 \\
\hline Iles Cooks (associé N-Z) ........... & -51827 & 16900 & -3067 \\
\hline Samoa américaines .......................... & -88025 & 46800 & -1881 \\
\hline 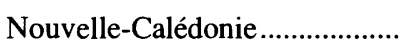 & -115140 & 167600 & -687 \\
\hline 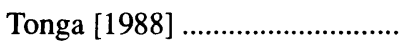 & -60134 & 96300 & -624 \\
\hline Samoa occidentales ..................... & -80472 & 157700 & -510 \\
\hline Tuvalu (autonome NZ) ............... & -5170 & 10200 & -507 \\
\hline Vanuatu & -59764 & 146400 & -408 \\
\hline 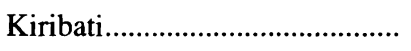 & -22161 & 71800 & -309 \\
\hline Fidji & -215321 & 725000 & -297 \\
\hline Papouasie/Nouvelle-Guinée ..... & -79695 & 3528500 & -23 \\
\hline
\end{tabular}

Source : Commission du Pacifique Sud.

De plus, ces pays reçoivent une bonne partie de leurs ressources des travailleurs émigrés : à Tokelau, les expatriés (3 300) en Nouvelle-Zélande et aux Samoa occidentales dépassent la population restée sur l'île (1600). Aux îles Cook également, on compte 24000 originaires en Nouvelle-Zélande contre 16900 dans l'île en 1982. On compte trois fois plus de Niueans résidant en Nouvelle-Zélande qu'à Niue. La plupart de ces îles sont en voie de dépopulation, frappée par un exode massif vers la Nouvelle-Zélande.

D'après une source récente ${ }^{1}$, en 1986 sur 499000 Polynésiens anglophones du Pacifique Sud, 185500 (dont 112000 Samoans) vivent en Australie, en Nouvelle-Zélande ou aux États-Unis. Compte tenu de l'immigration illégale, près de quatre Polynésiens anglophones sur dix vivent dans un pays industriel de la bordure du Pacifique.

Le tableau 2 montre l'ampleur du déficit extérieur par habitant, la faiblesse du taux de couverture, et l'importance l'émigration dans les îles étudiées par Bertram et Waters [1985].

Par ailleurs, le budget de fonctionnement de ces îles est alimenté surtout par l'aide de la Nouvelle-Zélande et de quelques autres pays donateurs, accordée sous forme de dons, et il sert principalement à employer une bureaucratie qui fournit la plupart des emplois. Ainsi l'administration distribue un pourcentage de la masse salariale totale qui va de $40 \%$ aux îles Cook, à $90 \%$ à Tokelau, en passant par $53 \%$ à Tuvalu, et $80 \%$ à Niue (Polynésie française : $55 \%$ en 1988). Tuvalu complète ces recettes par la vente de timbres aux philatélistes du monde

1. Geoffrey Hayes [1991], p. 1-58. 
Tableau 2. Déficit commercial et émigration dans les pays insulaires associés à la Nouvelle Zélande

\begin{tabular}{|c|c|c|c|}
\hline & $\begin{array}{c}\text { Taux de couverture } \\
\text { (tourisme inclus) } \\
1980-1983\end{array}$ & $\begin{array}{c}\text { Déficit extérieur par } \\
\text { habitant en } A \$ \\
(1983)\end{array}$ & $\begin{array}{l}\text { \% d'expatriés } \\
\text { dans les originai- } \\
\text { res de l'île } \\
\left(1986^{*}\right)\end{array}$ \\
\hline Iles Cook ……................... & 50 & 2501 & 66 \\
\hline Niue & 21 & 626 & 78 \\
\hline 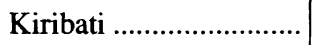 & 30 & nd & 4 \\
\hline Tokelau ............................... & 33 & 343 & 62 \\
\hline Tuvalu.............................. & 54 & 207 & 15 \\
\hline
\end{tabular}

Source : I.-G. Bertram et R.-F. Watters [1985], p. 497-519.

$(*)$ Sauf Kiribati et Tuvalu : 1981. Source pour les autres pays : Geoffrey Hayes [1991], p. 1-58.

entier. Les emplois créés dans l'administration permettent le développement du secteur tertiaire non marchand. Au total le secteur tertiaire marchand et non marchand fournit la majorité des emplois, de la même façon que dans les DOMTOM français.

Ces économies insulaires liées à la Nouvelle-Zélande ressemblent désormais en quelque sorte à des "banlieues dortoirs » de l'économie dominante, peuplées de retraités et de fonctionnaires. En effet, beaucoup d'émigrés reviennent prendre leur retraite dans leur île natale, et les relations familiales entre les émigrés et leur famille restée au pays peuvent subsister même sur plusieurs générations, entretenues par de fréquents voyages. Les réseaux de familles étendues se maintiennent malgré la grande dispersion de leurs membres, formant une «entreprise familiale multinationale » (transnational corporation of kin) ${ }^{1}$.

Le rôle essentiel joué par l'émigration et les envois de fonds des expatriés à leurs familles dans l'économie insulaire, et le développement limité observé dans ces îles amène à observer que le "secteur moderne " de ces économies se trouve en fait situé à l'extérieur, ce qui explique la faiblesse du développement productif. La logique de l'avantage comparatif dans le cadre d'une spécialisation internationale du travail amène l'économie insulaire périphérique à exporter directement ses travailleurs vers le «secteur moderne» situé dans l'économie «métropolitaine $»^{2}$ pour obtenir les marchandises convoitées, au lieu de chercher à les fabriquer sur place ou à exporter des marchandises pour les échanger contre celles de l'économie «métropolitaine ». L'effet d'éviction

1. G. Bertram [1986], p. 809-822.

2. Le terme « métropolitain » est utilisé ici au sens large, s'agissant d'un État « protecteur », quels que soient les liens politiques officiels (territoire, État indépendant " associé », État souverain bénéficiant d'accords économiques avec l'ancienne « métropole »). 
de la rente administrative et «familiale » (provenant de l'émigration) sur les activités productives est donc tout à fait vérifié dans ces économies insulaires ${ }^{1}$.

\section{L'économie MIRAGE dans les DOM}

L'économie des DOM présente beaucoup des caractères du modèle MIRAGE, avec notamment une très forte émigration.

La Réunion comptait 75000 personnes émigrées en métropole en 1982, et un taux de chômage de près de $40 \%$. En 1982, près de la moitié des jeunes de 25 à 29 ans nés à la Martinique résidait en France. Les autres avaient à faire face à un taux de chômage très élevé en Martinique.

De 1954 à 1982, le nombre de personnes nées dans les DOM-TOM et installées en France est passée de 24200 à 282300 (soit une multiplication par 11,7).

Autre caractère du modèle MIRAGE partagé indéniablement par les DOM : le rôle prépondérant des dépenses publiques d'origine métropolitaine dans l'emploi et le niveau de la demande, et un développement relativement faible du secteur productif moderne ${ }^{2}$.

Le modèle MIRAGE permet également d'analyser très utilement la situation de La Réunion :

" À la Réunion, le chômage était pratiquement inconnu au début des années soixante, du moins au sens où on l'entend aujourd'hui. À l'époque, on manquait plutôt de maind'œuvre pendant une grande partie de l'année, au moment de la campagne sucrière. Aujourd'hui, la Réunion est, de tous les départements français (y compris les autres DOM), celui où le taux de chômage est le plus important : $37 \%$. En raison du très fort ralentissement de l'émigration, l' essentiel de l'ajustement entre demande et offre d' emploi se réalise dans une augmentation massive du chômage ${ }^{3}$ ".

1. «An important feature of a MIRAB [MIRAGE] structure is thus the crowding-out of marginal productive activities by new opportunities to earn cash incomes in government employment and overseas. » I.-G. Bertram, R.-F. Watters [1985], p. 497-519.

2. Sans avoir eu connaissance, semble-t-il, des analyses de Bertram et Waters, C. de Miras [1987] à propos des DOM ou G. Blanchet [1989] à propos des TOM, font une analyse très proche de celle de ces deux auteurs, à propos de l'effet d'éviction, sur les activités productives, de la rente économique procurée par les transferts publics extérieurs. Ainsi, G. Blanchet remarque, à propos des TOM du Pacifique : « Le poids et le caractère non productif de ces dépenses [publiques métropolitaines] incitent moins au développement de la production qu'à l'acquisition immédiate auprès de la puissance de tutelle de biens qui renforcent la situation de dépendance. »

C. de Miras, pour sa part, note à propos de la Martinique : " Dans l'économie de transferts, l'industrie et l'ensemble des activités productives sont sapées dans leur ressort profond: la création de valeurs d'échange est inéluctablement évincée par les transferts publics et leur double, l'importation. "

3. Conseil économique et social, rapport de M. Guy Jarnac pour la préparation d'un avis sur « la situation économique et les conditions du développement des départements d'Outre-Mer », reproduit dans Problèmes économiques, $\mathrm{n}^{\circ} 2056,6$ janvier 1988. (Souligné par nous.) 
Dans ce DOM, la même politique qu'en Polynésie française, en plus accentuée du côté des prestations sociales (alignement partiel du SMIG et des prestations sociales, mais en plus indemnités de chômage et RMI - inexistants en Polynésie française), a considérablement ralenti l'émigration et dramatiquement développé le chômage, avec les conséquences récentes que l'on sait en 1991 (émeutes des jeunes du quartier du Chaudron). Analysé à partir du graphique 1, le tarissement de l'émigration et le développement corrélatif du chômage à La Réunion s'expliquent facilement : compte tenu des prestations sociales ajoutées au SMIG de La Réunion et d'un salaire «étranger » W* égal à (SMIG métropolitains + prestations sociales métropolitaines - coûts psychologiques et de transports), on est passé d'une situation (années soixante) où $\mathrm{W}_{0}<\mathrm{W}^{*}$ à une situation (actuelle) où $\mathrm{W}_{2}>\mathrm{W}^{*}$, pendant que la hausse de $\mathrm{W}_{g}$ se poursuivait régulièrement, largement au-dessus de $\mathrm{W}_{2}$ et de $\mathrm{W}_{0}$. On peut dire sans crainte de se tromper que la politique de «parité sociale » initiée par le gouvernement français a créé de toutes pièces le problème du chômage à La Réunion, en supprimant l'exutoire de l'émigration (provoquant au contraire une immigration métropolitaine significative), et en élevant artificiellement le niveau de productivité requis pour la création d'un emploi dans le secteur privé capitaliste. Contrastant avec ce chômage catastrophique à La Réunion, l'île Maurice, située non loin, possède une des densités de populations les plus élevées du monde ( $563 \mathrm{hab} / \mathrm{km} 2)$, mais les salaires y sont bien plus bas, faute de SMIG et de «parité sociale » financée de l'extérieur. L'île Maurice a supprimé le chômage, tombé à un taux de $4 \%$ en 1990, (contre $17 \%$ en 1968, au moment de l'indépendance), grâce au développement accéléré des emplois de sa zone franche industrielle d'exportation (croissance de $+30 \%$ par an pour le secteur industriel depuis 1984). La pénurie de main-d'œuvre y est aujourd'hui à l'ordre du jour.

Le rôle crucial du niveau de salaire " exogène » sur l'émigration et le chômage de l'économie insulaire peut être illustré par l'exemple des Samoa américaines : quand le salaire minimum a été fixé aux États-Unis à 4,25 \$ de l'heure en 1991, les fabriques américaines de thon en boîte installées dans l'île ont menacé de s'installer ailleurs si cette législation s'y appliquait aussi. Les représentants des Samoa américaines ont préféré renoncer à bénéficier du salaire minimum américain (pourtant inférieur à l'époque à celui de la Polynésie française : 4,72 \$ de l'heure). Il est vrai que la plupart des travailleurs de ces fabriques sont eux-mêmes des « immigrés » en provenance des Samoa occidentales, où le salaire est bien inférieur, les Samoans américains ayant pour leur part émigré en masse à Hawaï et sur la côte Ouest des États-Unis ${ }^{1}$. On peut néanmoins remarquer qu'il existe des cas où la «parité sociale »n'est pas forcément jugée souhaitable sur le plan économique et social, par les représentants des insulaires eux-mêmes, en raison des craintes concernant le développement du chômage.

1. David North, « Wages of Tuna Workers Frozen », Pacific Islands Monthly, 61 (7), 1991, p. 54-57. 


\section{L'économie «ARABE » en Polynésie française et dans les États fédérés de Micronésie}

Comme dans les autres DOM-TOM et les économies «MIRAGE » du Pacifique, la Polynésie française a une économie orientée presque exclusivement vers le secteur tertiaire, marchand (services privés, commerce, transports) et non marchand (administrations), financée par la rente administrative, dont la composante essentielle, dans le cas de la Polynésie française, est la rente «atomi-que » et les dépenses militaires associées, que représentent, en 1989, 59 milliards de F CFP pour un PIB de 281 milliards de F CFP. Le secteur tertiaire représente $73 \%$ du PIB en 1989 , contre $4,4 \%$ pour le secteur primaire (40 $\%$ en 1960 ), et $15,3 \%$ pour le secteur secondaire.

L'activité «productive» des secteurs primaire et secondaire a été largement « évincée " par la rente administrative qui a profité au secteur tertiaire marchand et non marchand : de 1962 à 1988, les emplois des administrations ont été multipliés par 7, ceux du secteur tertiaire marchand par 3,6, alors que les effectifs du secteur primaire baissent de $40 \%$. Les emplois créés dans le secteur secondaire ont augmenté moins vite que l'ensemble des emplois, et concernent surtout des secteurs protégés: l'énergie électrique, le bâtiment et les travaux publics (plus de la moitié des emplois du secteur secondaire), les industries de biens intermédiaires liées au bâtiment, les industries assurant la maintenance, l'entretien, la réparation de produits importés, et quelques industries de substitution d'importation (agro-alimentaire, ameublement, biens intermédiaires pour la construction), très fortement protégées, soit par des prohibitions d'importation (charcuterie, produits lactés hors CEE), soit par des contingentements, soit par des droits de douane prohibitifs (bière et boissons sans alcool, matériaux de construction, maisons en kit, meubles, matelas, habillement...). Enfin, de 1962 à 1988 , les emplois se sont accrus de $141 \%$, et $96 \%$ de cette hausse proviennent du secteur tertiaire marchand et non marchand.

Les exportations agricoles se sont effondrées depuis l'arrivée du CEP : la vanille, pour laquelle la Polynésie était un des plus grands exportateurs mondiaux, n'est pratiquement plus produite ni exportée. Le café, exportation importante naguère, est aujourd'hui importé, de même que les oranges, qui poussent pourtant encore à l'état sauvage sur les hauts plateaux de Tahiti. Seule la perle noire connaît un développement rapide à l'exportation. Elle présente, en effet, tous les caractères d'une ressource susceptible de fournir une "rente » naturelle, comme le phosphate ou le pétrole, en raison de sa rareté et du quasi-monopole mondial de la Polynésie française pour sa production, qui explique son prix moyen plus élevé que celui de la perle blanche de culture.

L'échec incontestable du développement touristique, malgré des atouts naturels indéniables, confirme également la théorie du dutch disease: avec 120000 touristes en 1991, la Polynésie française n'a pas accueilli plus de touristes qu'au début des années soixante-dix, et la baisse du nombre des touristes est constante depuis 1987. La Polynésie est classée par l'ONU septième destination touristique la plus chère du monde (sur 220) en décembre 1991. L'activité touristique, appartenant au secteur exposé, subit de plein fouet la hausse du coût 
Graphique 3. Répartition de la valeur ajoutée par secteur, Polynésie française, 1960-1984

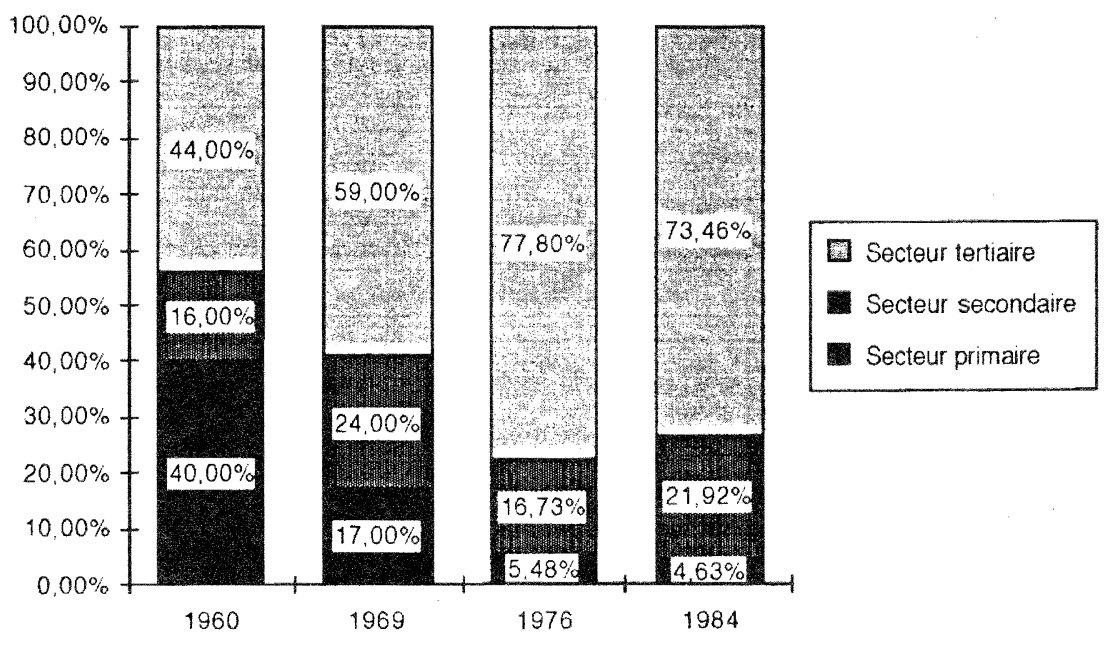

Source : Comptes économiques de la Polynésie française, 1960-1969 (INSEE) et 1976-1984 (ITSTAT).

Tableau 3. Évolution de la structure de l'emploi par secteur en Polynésie française

\begin{tabular}{l|r|r|r|r|r|r|r|r}
\hline & \multicolumn{2}{|c|}{1962} & \multicolumn{2}{c|}{1977} & \multicolumn{2}{c|}{1983} & \multicolumn{2}{c}{1988} \\
\hline & & $\%$ & & $\%$ & & $\%$ & & $\%$ \\
\hline Tertiaire non & & & & & & & & \\
marchand...... & 3264 & 12,31 & 14300 & 33,37 & 20648 & 35,68 & 23257 & 36,34 \\
Tertiaire & & & & & & & & \\
marchand & 5946 & 22,43 & 13900 & 32,44 & 18405 & 31,81 & 21862 & 34,16 \\
Secondaire & 5088 & 19,19 & 7500 & 17,50 & 10778 & 18,63 & 11334 & 17,71 \\
Primaire & 12211 & 46,06 & 7150 & 16,69 & 8032 & 13,88 & 7553 & 11,80 \\
\hline Total & 26509 & & 42850 & & 57863 & & 64006 & \\
\hline
\end{tabular}

Tableau 4. Actifs occupés en Polynésie française

\begin{tabular}{r|r|r|r|r|r}
\hline & \multicolumn{1}{|c|}{1962} & \multicolumn{1}{c|}{1977} & \multicolumn{1}{c|}{1983} & \multicolumn{1}{c}{1986} & \multicolumn{1}{c}{1988} \\
\hline Total des actifs occupés ........ & 26509 & 42850 & 57863 & 63003 & 64006 \\
dont administrations........... & 3264 & 14300 & 20648 & 22710 & 23257 \\
Variation annuelle moyenne.. & & $62-77$ & $77-83$ & $83-86$ & $86-88$ \\
Total des actifs occupés ........ & & 1089 & 3003 & 1713 & 502 \\
dont administrations........... & & 736 & 1270 & 687 & 274 \\
\hline
\end{tabular}

Source : Recensements de 1962, 1983, 1987, 1988.

E. Bruneau, « Emploi, net ralentissement depuis un an », Aspects, 3/4, août 1987, p. 37, ITSTAT. 
des ressources dans le secteur protégé (coût de la construction, de la main-d'œuvre, de la nourriture, de l'énergie, des télécommunications, des transports terrestres et aériens intérieurs). Les marges de ce secteur sont «pincées » par la hausse des coûts, la surévaluation du franc pacifique lié à un franc « fort », et la stabilité des prix des prestations exprimés en dollar.

Par rapport au modèle MIRAGE, en gros applicable sans grand changement aux DOM, et au petit territoire de Wallis-et-Futuna, quel est la spécificité de la Polynésie française ? L'absence d'émigration (c'est au contraire à une immigration non négligeable en provenance de métropole et d'autres DOM-TOM qu'on assiste jusqu'en 1986, la population née hors de Polynésie française représentant $13 \%$ de la population totale du territoire en 1988), et un chômage modéré, du moins jusqu'en 1988 (taux officiel en 1988 : 8,6 \% d'après le recensement).

\section{Tableau 5. Taux de chômage et migrations dans les DOM-TOM}

En \% de la population active

\begin{tabular}{l|l}
\hline Métropole [1986] ............. & 10,4 \\
Polynésie française [1988] & 8,6 (immigration nette (800/an) jusqu'en 1986 (-2 240)). \\
Guadeloupe [1986] ............ & 27,0 (note : dans ces trois départements). \\
Martinique [1986] ............. & 31,0 (très forte émigration vers la métropole) \\
Réunion [1988].................. & $\begin{array}{r}37,0 \text { (en 1982, plus d'une personne sur cinq née aux } \\
\text { Antilles vivait en métropole). }\end{array}$ \\
Nouvelle-Calédonie [1989] & 16,0 \\
Guyane [1986]................... & 22,0 \\
\hline
\end{tabular}

Source : ITSTAT, INSEE.

En effet, de 1954 à 1982, le nombre de personnes nées dans les DOM-TOM installées en France est passé de 24200 à 282 300. Parmi eux, un nombre négligeable de Polynésiens français, alors même que, selon G. Hayes ${ }^{1}, 40 \%$ des Polynésiens anglophones du Pacifique résident dans les pays industriels de la zone (Australie, Nouvelle-Zélande, États-Unis). Pourquoi cette absence d'émigration des Polynésiens français? Parce que le «secteur moderne à hauts salaires », vers lequel émigrent les Polynésiens insulaires du Pacifique, en Nouvelle-Zélande, en Australie, ou aux États-Unis (dont Hawaï), est venu à eux en Polynésie française, à travers le CEP et l'économie de service induite par sa présence. Les deux secteurs " moderne à hauts salaires » et "traditionnel convivial subventionné ", au lieu d'être situés l'un en métropole, l'autre sur place, coexistent en Polynésie française, l'un dans la zone urbaine autout de Papeete et

1. Hayes Geoffrey [1991], p. 1-58. Les Polynésiens anglophones sont originaires des îles : Cook, Niue, Tokelau, Tonga, Samoa occidental, Samoa US. Ils sont 499000 environ en 1986, dont $40 \%$ environ résident en Australie, en Nouvelle-Zélande ou aux ÉtatsUnis. 
sur les sites atomiques des Tuamotu, l'autre dans les districts éloignés et dans les autres îles de l'Archipel. C'est cette « société duale » qui rend l'émigration inutile : le complexe militaro-administratif français est à la porte du Tahitien ou du Paumotu. La rente atomique (RA) remplace les migrations (MI), de l'économie MIRAGE, on passe à l'économie ARABE.

La motivation pour l'émigration est, d'autre part, découragée par la forte création d'emplois publics dans les années soixante à quatre-vingt : le nombre de ces emplois administratifs est passé de 3264 en 1962 à 23257 en 1988, mais surtout par le niveau de leur rémunération, environ deux fois plus élevée qu'en métropole, sans aucun impôt sur le revenu ${ }^{1}$.

Il est pratiquement certain que le chômage réel est resté très faible en Polynésie française jusqu'en 1983, à cause d'un déplacement très rapide des courbes $\mathrm{L}_{g}$ et $\mathrm{L}_{m}$ vers la droite, en raison de l'effet multiplicateur des transferts publics civils et militaires dans le territoire, et cela malgré un très fort relèvement du SMIG de 1977 à 1983 (+ $100 \%$ ). En même temps, la politique d'aide aux archipels a certainement eu pour effet de relever le revenu du secteur de subsistance $\mathrm{W}_{1}$ : subvention du coprah, de la vanille, des engrais, des «travaux lourds » (labourages), régime de protection sociale en milieu rural, ce qui a permis de préserver dans les archipels le secteur traditionnel, et donc de limiter l'exode vers Tahiti, lequel a cessé entre 1977 et 1983, d'après les recensements de ces deux années.

L'existence d'un SMIG élevé (plus élevé qu'aux États-Unis) limite la capacité d'absorption en emplois du secteur privé, ${ }^{2}$ ainsi que la rentabilité des nouveaux investissements (notamment dans le tourisme ou dans la substitution d'importation). Il faut noter que le SMIG nominal stagne depuis 1989, après avoir augmenté très rapidement dans les années soixante-dix. Enfin, un SMIG plus élevé que dans les pays, développés ou non, de la région, et à peine moins élevé qu'en métropole, décourage l'émigration, ce qui enlève un exutoire, possible dans les autres îles, au problème du surplus de main-d'œuvre, et risque d'aggraver considérablement le problème du chômage.

1. Officiellement, les fonctionnaires d'État ont un traitement supérieur de $+84 \%$ (index de correction) par rapport au niveau métropolitain. En ce qui concerne les «agents contractuels » du Territoire et des communes, ils ont obtenu dans les années soixante-dix «l'alignement» sur les échelles de traitements des fonctionnaires « expatriés » à une époque où ces derniers bénéficiaient de salaires supérieurs de $113 \%$ au niveau métropolitain, puis ils ont obtenu l'indexation de leur traitement sur l'inflation locale, plus rapide que l'inflation métropolitaine jusqu'en 1986, ce qui a permis de maintenir, en pouvoir d'achat, un « index de correction » de 2,13 par rapport au niveau métropolitain, alors que celui des fonctionnaires d'État était abaissé à 1,84, puis partiellement indexé sur l'inflation métropolitaine, nettement moins rapide que l'inflation « locale » de 1980 à 1986.

2. Ce dernier est obligé de recourir à des méthodes très capitalistiques pour dégager une rentabilité, ce qui élève considérablement le coût en capital d'une création d'emploi dans le secteur industriel. 
Le modèle ARABE ne concerne pas que la Polynésie française. En effet, de nombreuses îles ont servi de base stratégique, soit pour des essais nucléaires ou balistiques, soit pour des forces plus conventionnelles (Pearl Harbor à Hawaï, Diego Garcia, Guam). L'économie de Guam et celle des "États fédérés de Micronésie » (anciens «Territoires sous tutelle (Trust Territories) des îles du Pacifique) est également fortement dépendante des activités militaires US. Après la guerre, les Américains utilisent plusieurs atolls pour des essais atomiques (Bikini, Eniwetoks) jusqu'en 1958, puis l'atoll de Kwajalein devient un centre d'essai des missiles anti-balistiques. Ainsi en Micronésie américaine :

«Les revenus domestiques couvrent 10 à $15 \%$ seulement des dépenses de fonctionnement des gouvernements, les crédits alloués par Washington subvenant au complément et à la quasi-totalité des investissements publics - soit 80 à $90 \%$ des investissements totaux. Le secteur public est le moteur de l'économie et emploie $55 \%$ des personnes salariées. Peu productif, le secteur privé consiste surtout en commerce d'importation et de distribution. La manne américaine permet de satisfaire les besoins d'importation croissants, passés, par exemple, pour les seuls États fédérés de Micronésie de 21 millions de $\$$ en 1977 à 49 millions de $\$$ en 1983 alors que, faute de surplus commercialisable, leurs exportations stagnent autour de 1,6 million $^{1}$. »

\section{LE DÉVELOPPEMENT PAR LA RENTE : UNE SPÉCIALISA- TION INTERNATIONALE LOGIQUE ET VIABLE À LONG TERME POUR LES PETITES ÉCONOMIES INSULAIRES?}

Rente atomique ou militaire (Polynésie française, Micronésie américaine), rente " phosphatière » (Kiribati, Nauru), rente administrative, aide internationale, envois de travailleurs émigrés, constituent les carburants essentiels des économies micronésiennes du Pacifique et des Antilles, conformes aux modèles ARABE ou MIRAGE. Faut-il s'en réjouir ou s'en désoler ? Le terme de rente a acquis une connotation négative de longue date. Bornons-nous à constater que le but de toute activité économique est d'obtenir le maximum de confort matériel avec le minimum d'efforts et de ressources. Jugé d'après ce critère, le mode de développement par la rente n'est pas plus mauvais qu'un autre, il est même le plus efficace sur ce plan, pourvu que la rente puisse être obtenue pour longtemps. Par ailleurs, l'origine de la rente se trouve dans la possession d'une ressource rare. Nul ne s'étonne de l'existence d'une rente pétrolière ou minière. La rente atomique ou administrative perçue dans les DOM-TOM ne devrait pas, $a$ priori, être vue différemment. Dans le cas des DOM-TOM, on peut même prétendre que la rente rémunère en fait une exportation non marchande vers la

\footnotetext{
1. J.-P. Reymondet-Commoy [1988].
} 
métropole, de sorte que l'échange apparemment déséquilibré ne l'est en fait pas du tout.

La rente et l'« échange inégal »: l'exemple de la Polynésie française

Le terme d' «échange inégal » est employé ici pour exprimer l'idée souvent émise que les DOM-TOM reçoivent en quelque sorte plus qu'ils ne donnent dans leurs échanges avec la métropole, «exploitant» ainsi le reste de la nation, ce qui constitue bien sûr un « échange inégal » dans le sens inverse de celui dont parlait A. Emmanuel dans son célèbre livre.

Prenons l'exemple de la Polynésie française : elle n'a pas de pétrole, mais elle peut échanger les biens et services de la métropole contre un « service non marchand » précieux : la " sécurité nationale par la dissuasion nucléaire ». Chaque année, la valeur des exportations « invisibles » de ce service non marchand vers la métropole représente une somme colossale, à savoir ce que la métropole dépense (sur le territoire ou en métropole) pour fabriquer ce «bien public » sur le territoire : 59 milliards de F CFP en 1990. Or, la même année, les importations du territoire étaient de 91 milliards et les exportations «visibles» de 11 milliards de F CFP, soit un taux de couverture (exportations/importations) de $12 \%$ seulement. En rajoutant les «exportations» de services (tourisme) : 16 milliards de F CFP et celles de «dissuasion nucléaire » : 59 milliards de F CFP, on obtient un total de $11+16+59=86$ milliards de F CFP, ce qui donne un taux de couverture de $86 / 91=94,5 \%$.

À $5 \%$ près, donc, les échanges « visibles » et « invisibles » du territoire sont équilibrés. Ces exportations « invisibles » n'en sont pas moins bien réelles, et précieuses pour la France, sinon pourquoi dépenserait-elle tant pour les obtenir?

Il faut donc mettre en perspective la notion, à connotation péjorative, de « rente » perçue par les DOM-TOM « au détriment » du contribuable métropolitain. Que la contribution «productive» des DOM-TOM paraisse faible en regard de leur niveau de consommation « marchande " ne doit pas faire oublier leur contribution «non marchande » (géostratégique) et culturelle à l'ensemble de la nation.

\section{La théorie de l'avantage comparatif élargie aux échanges non marchands}

En élargissant quelque peu la «théorie des avantages comparatifs » au domaine des services publics non marchands, on arrive à dégager la rationalité profonde qui préside au choix de l'économie de rente et de la «spécialisation »

1. Tous les chiffres sont tirés du rapport annuel de l'IEOM en Polynésie française. 
définitive de ces pays insulaires dans l'aide, la bureaucratie et (si nécessaire) l'exportation de travailleurs émigrés, aux dépens de l'activité productive.

Économiquement, on peut dire que l'échange de «services», marchands (salaires contre travail à l'étranger) et non marchands (aide publique contre allégeance politique et exportation de services géostratégiques ou militaires), qui s'instaure entre la microéconomie insulaire et la métropole, permet d'obtenir des combinaisons du bien public «mode de vie convivial traditionnel » et du bien privé «produits manufacturés » qu'il serait impossible d'obtenir en l'absence de cette forme d'échange. Mieux vaut alors renoncer à améliorer la productivité du travail dans la production de biens et services privés pour continuer à bénéficier de la meilleure efficacité dans la production du bien public socioculturel tout en obtenant en échange du bien "géostratégique » (public non marchand) des biens marchands produits dans l'économie métropolitaine : on gagne alors «sur les deux tableaux » : meilleure productivité du temps de loisir dans la production de « qualité de vie » insulaire, et meilleur niveau de vie insulaire permis par la haute productivité de travail dans l'économie «métropolitaine » : si l'on concevait la «frontière des possibilités de production » de l'économie insulaire et de l'économie métropolitaine dans l'espace des biens marchands et non marchands, privés et publics, on pourrait montrer que l'échange avec l'économie métropolitaine permet d'atteindre une frontière des possibilités de consommation plus élevée pour les deux pays associés, permettant des combinaisons de biens marchands privés et de bien public non marchand (socioculturel d'une part, géopolitique et stratégique d'autre part) plus avantageuses pour l'économie micronésienne comme pour l'économie «métropolitaine ». L'explication en est simple : si l'utilisation géostratégique de l'île permet d'y obtenir un résultat semblable avec une meilleure productivité (par exemple, l'île peut permettre d'économiser un porte-avions atomique, où l'expérimentation nucléaire permet de consacrer moins d'argent aux forces conventionnelles), alors l'économie de ressources dégagée par le grand pays lui permet de se déplacer le long de sa «frontière des possibilités de production » vers une plus grande production de biens marchands civils. Ce gain peut être en partie reversé aux habitants de l'île sous forme d'une « rente » qui leur permet d'importer ces biens marchands civils sans contrepartie en exportations « civiles » vers l'extérieur ${ }^{1}$.

Qu'une petite économie insulaire soit plus efficace dans la production de «qualité de vie » à partir d'une quantité de loisir donnée, ne devrait pas être contestable dans la plupart des îles tropicales. S'il fallait une preuve de cette assertion, elle serait fournie par les sommes d'argent élevées que les touristes du pays métropolitain sont disposés à dépenser juste pour avoir le privilège de venir passer leur temps de loisir dans l'île «paradisiaque » plutôt que de passer

1. Pour une démonstration géométrique rigoureuse de ce point, cf. Bernard Poirine, «Théorie de l'échange international non marchand : l'économie de rente ou de transfert dans les petites économies insulaires », Table ronde juridique sur la Polynésie française, Université française du Pacifique, mai 1993. 
ces vacances chez eux. Elle serait confirmée également par les efforts coûteux que produit le Club Méditerranée pour reproduire « artificiellement» ce «bien public socioculturel» que constitue une société conviviale polynésienne "synthétique » dans tous ses villages autour du monde, et qui font le succès de sa formule de « village ». Que l'économie insulaire ait également un «avantage comparatif» dans l'exportation du service public non marchand "géostratégique " se conçoit aisément : les îles et archipels possèdent une «zone économique " très étendue, elles sont des «porte-avions » et des «escales » naturelles pouvant servir à " quadriller » le globe ou une partie du globe, et, enfin, leur faible population limite le coût pour l'économie métropolitaine d'obtenir en échange leur collaboration politique, stratégique et culturelle.

Dans la théorie du commerce international, chaque pays améliore son niveau de vie en se spécialisant dans les productions pour lesquelles il possède un avantage comparatif, et en important les autres. Ici, la petite économie insulaire se spécialise dans la production de biens publics : « mode de vie convivial traditionnel à basse productivité » (à usage intérieur) et «position géostratégique » (à usage d'exportation). Éventuellement, si nécessaire (en cas de pression démographique très forte et de créations d'emplois insuffisantes), elle exportera également des «services de travailleurs émigrés à l'étranger ». Elle obtient en échange les produits manufacturés du pays à haute productivité, financés par les transferts publics et (éventuellement) les remises de travailleurs émigrés. Cela explique pourquoi les activités du secteur «productif » sont " évincées » par les transferts publics: cela ne correspond pas à l'avantage comparatif dans l'échange, ce sont donc des productions abandonnées au profit de celles, non marchandes ou de services, pour lesquelles l'avantage comparatif existe. Cette stratégie de développement baptisée ici MIRAGE et ARABE apparaît donc en définitive parfaitement logique dans le cadre d'une théorie élargie de l'échange international, car elle correspond à une spécialisation optimale des petites économies insulaires.

\section{Développement dépendant ou développement dans l'interdépen- dance?}

Si l'on tient compte de la volonté de la métropole d'intégrer toujours plus étroitement, du point de vue du niveau de vie et des avantages sociaux, les DOM-TOM à l'ensemble national, y a-t-il même un sens à parler de dépendance économique, est-il même pertinent de calculer une balance des échanges avec la métropole et d' utiliser les mêmes critères de jugement que pour une économie ayant vocation à devenir indépendante, à moins d'admettre implicitement comme une évidence la perspective inéluctable ou tout au moins souhaitable d' une indépendance politique dans un avenir proche?

On ne songerait pas à calculer, par exemple, la balance des échanges d'une « ville de garnison » métropolitaine avec le reste de la nation, encore moins à savoir si la contribution de ses habitants aux activités «productives » dans l'agriculture et l'industrie est suffisante pour que cette ville présente les caractè- 
res d'une économie « viable », « équilibrée », « productive », « autonome », ou tout autre qualificatif fortement teinté, qu'on le veuille ou non; de jugement de valeur ou d'a priori idéologique et politique. L'a priori en question consiste à faire semblant de ne pas voir que l'apport d'une collectivité locale à la nation ne se mesure pas uniquement en termes de valeur ajoutée " productive », mais plus largement en termes culturel, ou géostratégique, par exemple. Personne ne songe à penser que les habitants d'une "ville de garnison " métropolitaine sont « assistés » ou « improductifs » parce qu'il n'y a pas d'usine ou de paysans à cet endroit, mais beaucoup de commerçants et autres fournisseurs de services « improductifs », entretenus par la « rente militaire » que leur accorde le reste de la nation. Tout le monde s'accorde à penser, au contraire, que ces habitants apportent une contribution "normale » à la nation. En revanche, la contribution géostratégique des DOM-TOM, et singulièrement de la Polynésie française, semble mal perçue, si bien qu'on parle plus naturellement de rente, de transfert ou de «danseuse de la nation » qu'à propos de n'importe quelle «ville de garnison » en métropole. En fait, la différence d'optique ne peut être justifiée que si l'on se place a priori, soit dans une perspective colonialiste traditionnelle (les DOM-TOM n'étant pas alors considérés comme partie intégrante de la nation), soit dans une perspective indépendantiste (pour la même raison), alors qu'on devrait se placer, du strict point de vue du statut politique actuel des DOM-TOM, tout simplement du point de vue de l'observation d'un sousensemble économique pleinement intégré à un ensemble plus vaste, qui trouve spontanément un équilibre adapté au rôle qui lui est dévolu au sein de cet ensemble, la "rente économique "étant la simple contrepartie d'un apport géo-stratégique et culturel.

Autrement dit, la Polynésie française et les autres DOM-TOM auraient une « spécialisation » dans l'ensemble français, semblable à celle d'une ville de garnison ou de retraités de l'hexagone, rôle qui justifierait le caractère « déséquilibré » de leur économie.

Seule la volonté de placer un département ou territoire sur un plan différent des autres collectivités de la République (position néo-coloniale), ou bien de le placer délibérément dans une perspective d'indépendance à plus ou moins long terme, semble justifier la position de considérer l'économie " tertiaire » engendrée par les transferts métropolitains comme le symptôme d' un dysfonctionnement économique majeur.

\section{CONCLUSION. ÉCONOMIES DÉSÉQUILIBRÉES, OU ÉCONOMIES ADAPTÉES ?}

Toutes les économies de rente présentent tous les symptômes de graves déséquilibres économiques, d'après les critères habituels. Mais peut-on véritablement parler alors de déséquilibres? En fait, c'est vers un nouvel équilibre, 
parfaitement adapté et rationnel, que se dirigent ces économies. Là où produire n'est plus nécessaire pour consommer, où la croissance du niveau de vie paraît de plus en plus déconnectée des progrès de productivité, où la contrainte d'équilibre externe n'est pas un problème, aucun motif n'exige que « les grands équilibres macro-économiques » traditionnels soient respectés. Paradoxalement, le déséquilibre entre la demande et l'offre locale générateur de déficit extérieur est une conséquence logique de la croissance impulsée par la demande publique dans une "économie de transfert ", et non pas un symptôme de déséquilibre économique susceptible de remettre en cause la croissance.

Devant ce constat, on peut réagir de deux façons : le déplorer, pour des raisons « morales ", et chercher à en sortir, par des mesures visant à promouvoir une plus grande autonomie économique, ou bien trouver le meilleur moyen de perpétuer la rente en question en essayant d'en accroître le plus possible l'effet multiplicateur sur l'activité locale.

- La première position recherche les voies d'une plus grande autonomie économique par l'accent mis sur le développement des secteurs « productifs » (primaire et tertiaire), par la recherche de la compétitivité des produits locaux, par des subventions accordées aux secteurs productifs, par une défiscalisation des investissements métropolitains dans les secteurs « productifs » des DOM-TOM, etc.

- La deuxième position fait le constat de l'échec de la plupart des tentatives visant à susciter un développement du secteur productif, que ce soit dans l'agriculture, dans l'industrie, ou dans le tourisme, en raison de l' "effet d'éviction » qu'exerce la rente distribuée par l'administration sur les activités du secteur « non protégé » de l'économie. De là à soutenir que l'économie de rente est un état durable et même préférable au développement du secteur productif, il n'y a qu'un pas que n'hésite pas à franchir G. Bertram en ce qui concerne les petites économies insulaires indépendantes du Pacifique :

« Le problème économique essentiel pour un grand nombre de très petites économies insulaires du Pacifique est la préservation et l'accentuation de leur statut de société rentière, et des niveaux de vie relativement élevés qui l'accompagne. La préférence marquée par les plans de développement régionaux pour une croissance " auto-entretenue " (sustainable) des activités productives n'atteint pas son but dans ces petites économies. Ce n'est pas l'activité productive, mais les revenus de rente qui doivent être soutenus et perpétués au cours du temps ${ }^{1}$. »

Dans cette optique, on ne peut parler de "mal développement »ou de déséquilibre, mais plutôt d' une économie intégrée à dessein dans un ensemble plus vaste, ayant trouvé spontanément un équilibre adapté à la fonction spécialisée qui lui est dévolue à la périphérie de cet ensemble (fonction géostratégique, vitrine culturelle de la France dans un ensemble régional anglo-saxon, et terrain

1. Traduit de I.-G Bertram [1986], p. 809. 
d'essai de la force de frappe dans le cas de la Polynésie française); et gérant au mieux, c'est-à-dire avec le moindre effort possible la "rente " accordée en contrepartie de sa contribution volontaire à la collectivité nationale. Ainsi ce serait faire un mauvais procès que d'affirmer que les nombreux travailleurs polynésiens ou métropolitains occupés directement ou indirectement par l'armée et le Centre d'expérimentation du Pacifique ( $23 \%$ de la masse salariale en 1984), sont des travailleurs «improductifs », et d'en conclure que le déficit commercial et la sous-industrialisation de la Polynésie française reflètent une capacité productive insuffisante de l'économie polynésienne, alors même que cette orientation vers des tâches « improductives » de la plus grande partie de la main-d'œuvre est le résultat d'une stratégie consciente de la métropole pour «spécialiser » la Polynésie dans ce rôle de base atomique. L'orientation de l'économie vers des tâches « improductives » a été voulue par l'État français, qui n'a pas lésiné sur les moyens pour attirer les jeunes agriculteurs ou pêcheurs vers les chantiers du CEP, puis vers l'administration, tout en provoquant par le brusque afflux d'immigrants à haut pouvoir d'achat une demande qui ne pouvait, ni en quantité, ni surtout en qualité, être satisfaite par le tissu productif local. Les exportations traditionnelles (café, vanille, coprah, nacre) ont été «évincées » par une exportation «non marchande » d'un service public géostratégique et militaire vers la métropole.

Si la rhétorique de l'indépendance économique est omniprésente au niveau des discours locaux dans les DOM-TOM, la volonté politique de "sevrer" réellement l'économie des transferts extérieurs n'existe pas vraiment. Le discours dominant en Polynésie française, illustré par le récent «pacte de Progrès ", par exemple, semble plutôt se résumer à une proposition du genre : «La rente (atomique ou militaire) nous a rendu dépendants, il faut maintenant lui substituer une rente civile destinée à nous aider à développer notre autonomie économique. " Que personne ne semble saisir le caractère paradoxal de cette proposition (réclamer plus de rente pour réduire la dépendance envers la rente), semble révélateur d'un état d'esprit bien enraciné à la suite de trois décennies d'économie de rente. Comme le remarque Claude de Miras ${ }^{1}$ à propos de la Martinique, on peut se demander dans quelle mesure la constante référence à l'objectif d' indépendance économique par le développement de la production locale ne remplit pas plutôt une fonction idéologique, celle de rendre plus supportable le « coût psychologique », en termes de dignité, de ce qui est perçu (à tort, nous l'avons montré), comme de l'« assistanat généralisé ».

L'indépendance politique ne signifie pas la disparition de l'économie de rente, car c'est le mode de développement prépondérant dans la plupart des micro-économies insulaires indépendantes ou autonomes du Pacifique et de la Caraïbe, la rente prenant simplement d'autres formes avec l'indépendance (aide budgétaire étrangère, remises de travailleurs émigrés, exploitation de phosphates, accords de pêches, bases militaires étrangères). De ce point de vue,

1. Claude De Miras [1987], p. 399-417. 
la Polynésie française, par exemple, a un mode de développement qui ne diffère des micro-États insulaires indépendants ou autonomes du Pacifique que sur deux points : d'une part, le niveau exceptionnellement élevé de la rente et du niveau de vie induit, et d'autre part, l'inexistence de l'émigration (courante avant l'arrivée du CEP), due au fait que le CEP a apporté sur place le haut niveau de vie que les insulaires d'autres parties du Pacifique vont chercher dans les métropoles régionales à économie hautement productive (Nouvelle Zélande, Australie, États-Unis).

Mises à part ces différences, on peut appliquer les mêmes conclusions que les microéconomies insulaires soient indépendantes, "associées", dépendantes « autonomes », ou "départements » : le développement dans l'interdépendance avec une puissance industrielle est un choix viable, logique et justifié. Si sur le plan politique, plusieurs formules d'interdépendance s'offrent, limitant plus ou moins sévèrement la souveraineté insulaire « périphérique » par rapport au centre industriel, les avantages économiques de l'interdépendance acceptée sont en général réciproques et mutuellement avantageux pour les deux parties, si l'on tient compte de l'aspect non marchand de l'échange.

Un prochain article développera plus rigoureusement la théorie de l'échange international non marchand et discutera plus en détail la viabilité à long terme du mode de développement par la rente pour les petites économies insulaires.

\section{RÉFÉRENCES BIBLIOGRAPHIQUES}

ALAM S. [1982], «The Basic Macro-economics of Oil Economies», The Journal of Development Studies, 18 (2), janvier, p. 205-216.

ANTHEAUME B. et BONNEMAISON J. [1988], Atlas des Iles et États du Pacifique Sud, GIP RECLUS/PUBLISUD.

ASSOCIATION POUR LA RECHERCHE ET LA TECHNOLOGIE À LA RÉUNION [1988], Les économies insulaires: stratégie de développement des économies insulaires à pouvoir d'achat élevé. Actes du colloque de Saint-Denis de La Réunion, 7-10 novembre 1988.

BerTRAm I.-G. [1986], « Sustainable Development in Pacific Micro-Economies », Word Development, 14 (7), p. 809-822.

BerTRAM I.-G., WaTters R.-F. [1985], "The MIRAB Economy in South Pacific Microstates », Pacific Viewpoint, 26 (3).

BERTRAM I.-G., WATTERS R.-F. [1986], «The MIRAB Process : Some Earlier Analysis and Context », Pacific Viewpoint, 27 (1) (mai), p. 47-57.

BLANCHET G. [1984], L'économie de la Polynésie française de 1960 à 1980, Travaux et documents $\mathrm{n}^{\circ} 10$, Orstom, Tahiti.

Blanchet G. [1985], L'économie de la Polynésie française de 1960 à 1980, Travaux et documents $n^{\circ} 195$, Éditions de l'ORSTOM, Paris. 
BLANCHET G. [1987], Croissance induite et développement autocentré en Polynésie française, thèse de doctorat d'État de sciences économiques, Université de Rennes I, 1986, Travaux et documents microédités, Éditions de l'ORSTOM, Paris.

BLANCHET G. [1989], « Du discours à la réalité en milieu insulaire : rhétorique du développement autocentré et pratique du développement intégré », Journal de la société des océanistes, 88-89, 89 (1 \& 2), p. 45-51.

BrunEau É. [1988], Comptes économiques de la Polynésie française, 1976-1984, Dossier 13, Institut territorial de la statistique, Papeete, Tahiti.

CAMPAN E., GRIMAUD A. [1989], « Le syndrome hollandais », Revue d'économie politique, 6.

ConNELl J. [1988], « Sovereignty \& Survival, Island Microstates in the Third World », Research Monograph, 3, University of Sydney.

$\rightarrow$ CORDEN M., NEARY J.-P. [1982], « Booming Sector and De-Industrialisation in a Small Open Economy », Economic Journal, 92, décembre, p. 825-848.

DE MIRAS C. [1988], « L'économie martiniquaise : croissance ou excroissance ? », Tiers Monde, 29 (114).

DE MIRAS C. [1987], "L'économie martiniquaise : développement sans croissance », dans Iles tropicales : Insularité, « insularismes », coll. «Iles et archipels », $\mathrm{n}^{\circ} 8$, édité par le Centre de recherche sur les espaces tropicaux, p. 399-417.

HAYES G. [1991], "Migration, Metascience, and Development Policy in Island Polynesia ", The Contemporary Pacific, 3 (1), printemps, p. 1-58.

HEIN P. [1988], "Problems of Small Island Economies », dans CRUSOL Jean, HEIN Philippe, Vellas François (dir.), L'enjeu des petites économies insulaires, Paris, Economica, p. 1-42.

PoIRINe B. [1991], L'économie de la Polynésie française : de la rente au développement, thèse de doctorat de sciences économiques, Université Paris II, 29 octobre 1991, 893 pages.

REYMONDET-COMMOY J.-P. [1988], « La libre association avec les États-Unis comme stratégie de développement de la micronésie », colloque, Stratégies de développement des économies insulaires à pouvoir d'achat élevé, Saint-Denis de La Réunion, 7-10 novembre 1988, Région Réunion.

CONSEIL ÉCONOMIQUE ET SOCIAL [1988], Rapport de Guy Jarnac pour la préparation d'un avis sur « la situation économique et les conditions du développement des départements d'Outre Mer », reproduit dans Problèmes économiques, 2056, 6 janvier 1988. 